\title{
Zur Herkunft von finn. runo 'Lied'
}

Seit V. Thomsens epochemachender Abhandlung "Den gotiske sprogklasses indflydelse pả den finske" vom Jahre 1869 (deutsch von E. Sievers 1870, jetzt Saml. Afhandl. II, 212) fassen nahezu alle Forscher sowohl auf fennologischer wie auf germanistischer Seite das finnische Wort runo 'Lied' als Entlehnung von urgerm. *rünō 'Geheimnis' auf, so noch - nach freundlicher Mitteilung von Professor E. Itkonen - in dem zur Zeit im Druck befindlichen Bd. IV von Suomen kielen etymologinen sanakirja.

Freilich hat man bei dieser Etymologie längst zwei Unsicherheitsfaktoren mit in Kauf genommen, nämlich einmal den kurzen Wurzelvokal des finnischen Wortes gegenüber dem $-\bar{u}$ - des germanischen Etymons, sodann die stark verschiedenen Bedeutungen des finnischen und des germanischen Wortes.

In der Tat ist der kurze Wurzelvokal von finn. runo auffallend. V. Thomsen führt in "Indflydelse" 45 (= "Einfluss" $51=$ Saml. Afh. II, 97) einige germanische Lehnwörter mit $-\bar{u}-$ an, die auch in der finnischen Entlehnung -uu- beibehalten haben, z.B. muuri 'Mauer', ruuni 'braun', suutari 'Schuhmacher'. Aber in diesen Fällen handelt es sich offenbar um sehr junge Entlehnungen. Andererseits weist auch finn. rikas 'reich' kurzen Stammvokal gegenüber urgerm. ${ }^{*}$ rikkiaz und den verwandten Wörtern der uns überlieferten germanischen Sprachen auf, so dass der kurze Wurzelvokal von finn. runo nicht unbedingt gegen die etymologische Verknüpfung mit urgerm. ${ }^{*} r u \bar{n} \overline{\mathrm{zu}}$ sprechen braucht.

Im übrigen hat es mit finn. rikas - auch abgesehen von der Kürze des Wurzelvokals - seine eigene Bewandtnis: urgerm. ${ }^{*}$ rikiaz hatte zweifellos nur die Bedeutung 'mächtig', während das finn. Wort nur 'reich' bedeutet, dagegen valtava und mahtava 'mächtig'. Noch im Alt- 
nordischen bedeutet rikr (Akk. Sg.m. rikjan und mit Ubergang in die $a$-Klasse rikan) ausschliesslich 'mächtig', insbesondere 'politisch mächtig'. Das gleiche gilt von ags. rice. Nur auf deutschem Boden erhält dieses Etymon frühzeitig neben 'mächtig' auch die Bedeutung 'reich', z.B. as. rīki. Es erscheint mir daher durchaus möglich, dass finnisch rikas eben wegen seiner Bedeutung 'reich' erst ein verhältnismässig spätes Lehnwort ist. Man hat ja längst beobachtet, dass die ursprünglich einer ur- oder altgermanischen Endung entsprechenden Wortausgänge im Finnischen gelegentlich als nunmehr bedeutungslose Suffixe willkürlich übertragen werden konnten, so dass etwa -as nicht ohne weiteres aus einem urgermanischen Wort mit dem Ausgang -as bzw. - $a z$ des Nom. Sg.m. hervorgegangen zu sein braucht.

Schwererwiegend ist der Unterschied in der Bedeutung. Nach dem "Nykysuomen sanakirja»" hat runo nur die folgenden drei Bedeutungen: 1. Ein gewöhnlich kurzes literarisches Erzeugnis, das ein bestimmtes rhythmisches oder metrisches Schema befolgt oder in der Redeform einem solchen Schema ähnelt, vergleichbar mit laulu 'Lied', virsi 'religiöses Lied'. - 2. Eine Darbietung in Gedichtform. - 3. In der Volksdichtung = runon laulaja 'Sänger'. Vgl. dazu auch Hakulinen, "Suomen kielen rakenne ja kehitys" ("Bau und Entwicklung der finnischen Sprache»), 3. Aufl. 1967, 286.

Es muss also ausdrücklich hervorgehoben werden, dass runo auch in der heutigen finnischen Umgangssprache immer nur einen poetischen, keinen prosaischen Text oder Textabschnitt bezeichnet.

Das Wort runo ist auch in weiteren Kreisen ausserhalb Finnlands besonders durch seine Verwendung im Kalevala bekannt.

Die von E. Lönnrot aus vielen Einzelstücken zusammengesetzte, sozusagen klassische Form des Kalevala ist in 50 runoja 'Gesänge' eingeteilt, und hierbei entspricht das Wort runo genau der ersten im Nykys. san. angegebenen Bedeutung (s.o.), indem es etwa je einem der 24 Gesänge der llias und der Odyssee gleichkommt, also ein im Verhältnis zum ganzen kurzes Teilstück einer Dichtung bezeichnet. Dabei haben die einzelnen runot - wie die Gesänge Homers - verschiedenen Umfang.

${ }^{1}$ In allen finnischen Partien dieses Aufsatzes durfte ich mich der Hilfe von Prof. G. J. Stipa erfreuen. 
Innerhalb des eigentlichen Textes des Kalevala findet sich runo sowohl in der Bedeutung 'Lied' wie in der von 'Sänger' (vgl. Turunen, "Kalevalan sanakirja» s.v.). Dabei möchte ich es für wahrscheinlich halten, dass die Bedeutung 'Lied' die typologisch primäre ist; man vergleiche an. skáld n., das ursprünglich wohl 'Scheltlied' bedeutete (vgl. zuletzt K. v. See, GRM, 1964, 11), später, unter Beibehaltung des neutralen Genus, 'Dichter, Skalde'; an. ó $\Varangle r$ (alter $u$-St.) 'Erregung, dichterische Begeisterung, Dichtung', kymr. gwawd 'Gedicht' : air. fáith 'Dichter', lat. vātes 'Prophet'.

Für die Bedeutung von runo im Text des Kalevala seien hier nur wenige Proben aus dem 12. Gesang zitiert. Charakteristisch ist dabei die Verwendung von endreimenden Synonymen an den Versschlüssen:

$12,389 \mathrm{ff}$.:

Kuuli ulkoa runoja, läpi sammalen sanoja,

Läpi seinän soittajoita, läpi lauan laulajoita.

'Er hörte von draussen Lieder (in der Hütte), durch das Moos Worte, durch die Wand Musizierende, durch die Bretter Sänger'. In diesem Beispiel ist runoja durch das bedeutungsverwandte sanoja eindeutig als Nomen actionis 'Lied' bestimmt, während in der zweiten Hälfte sich zwei Nomina agentis gegenüberstehen.

12, 401: Lauloivat Lapin runoja 'Sie sangen lappische Lieder'. Dagegen 12, 455 f.:

Parahille laulajille, taitavimmille runoille.

'Den besten Sängern, den geschicktesten Liedersängern'.

Zwar treten im Kalevala als wichtige Personen Zauberer auf, aber die einzelnen runot an sich sind keinesfalls Zauberlieder, sondern schlechthin Lieder der Volksdichtung, so dass also in dem Worte runo selbst nichts auf Zauber oder Geheimnis deutet. Gewiss könnte man sich auf die Annahme berufen, dass uns runo eben nur aus jüngerer Zeit bekannt ist, und sich in der Bedeutung 'Lied' erst im Lauf eines guten Jahrtausends aus einer älteren Bedeutung 'Zauberlied' oder 'Zauberspruch' entwickelt habe; aber eine solche Annahme wäre eben nur eine völlig unbeweisbare Theorie. 
Das Wort runo findet sich im übrigen ausschliesslich im Finnischen (und Karelischen), in keinem anderen finnischugrischen Idiom, nicht einmal in den anderen ostseefinnischen Sprachen.

Hier sei eingefügt, dass ein in den lappischen Dialekten auftretendes Etymon rudn(a) 'Murmeln, Lärm, Summen' nach $\mathrm{V}$. Thomsen, "Berøringer mellem de finske og de baltiske (litauisk-lettiske) sprog", Kph. 1890, 214 (= Saml. Afh. IV, 369) keine unmittelbare Verbindung mit finn. runo hat, sondern wohl aus einer neuskandinavischen Sprache entlehnt ist; vgl. etwa norw. dial. und schwed. runa 'heimlich miteinander sprechen'; über die lappischen Wörter ausführlich J. K. Qvigstad, "Nordische Lehnwörter im Lappischen", Christiania 1893, 272.

In diesem Zusammenhang mag auch lett. runa (mit kurzem Wurzelvokal) erwähnt werden, dessen Bedeutung sich in dem Feld 'Rede, Gespräch, Unterhaltung, scherzhafte, aber auch geheime Unterhaltung' bewegt. In dem Wörterbuch von Mühlenbach-Endzelin wird eben wegen der Kürze des -u- eine Entlehnung aus dem Germanischen abgelehnt, jedoch keine positive Erklärung gegeben. Schon früher hatte sich V. Thomsen in den "Berøringer» 213 (Saml. Afh. IV, 368) mit diesem lettischen Wort beschäftigt und es für eine Entlehnung aus dem Gotischen, vielleicht auch erst aus dem Deutschen oder Nordischen gehalten. Mir ist die Entlehnung aus einem verhältnismässig späten Stadium des Deutschen am wahrscheinlichsten, wenn auch dabei der kurze Wurzelvokal unerklärt bleibt. Es ist auch zu bemerken, dass lett. runa nebst dem Verbum runät innerhalb der baltischen Sprachfamilie isoliert dasteht.

Was nun die germanische Wortsippe *rūnō- betrifft, so hat man finn. runo wegen seiner Endung als Entlehnung aus urgerm. * $r u \bar{n} \bar{o}$ (N.Sg.) angesehen. Die Grundbedeutung dieses germanischen Wortes liegt eindeutig in got. rūna 'Geheimnis'

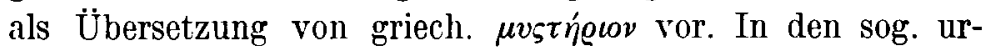
nordischen Runeninschriften ${ }^{1}$ tritt dieses Etymon zufrühest (2. H. des 4. Jh.s) auf dem ostnorwegischen Stein von Einang

1 Die urnordischen Runeninschriften werden im folgenden nach den Nummern des Buches "Die Runeninschriften im älteren Futhark" von W. Krause mit Beiträgen von H. Jankuhn, 1966, (abgekürzt KJ) zitiert. 
(KJ 63) auf: [ek Goldagastik rūnō faihidō 'Ich G. malte (oder: schrieb) die Rune'. Der Singular wird hier, aus der ursprünglichen Bedeutung 'Geheimnis' entwickelt, im kollektiven Sinn verwendet; ebenso (aber erst gegen 600) im Anfang der Inschrift des westgötischen Steines von Noleby (KJ 67): rūnō fāhi raginaku(n)dō 'Rune(n) male ich, von den Ratern (Göttern) stammende'. In anderen urnordischen und stets in den wikingerzeitlichen Runeninschriften zeigt das Wort auch formal pluralische Endungen.

In der klassisch-altnordischen Literatur bewegt sich rún in dem Bedeutungsfeld 'Geheimnis', 'geheime oder vertrauliche Unterhaltung', 'Rune'. Es erübrigt sich hier, dafür Beispiele anzuführen, weil man sie leicht in jedem Wörterbuch des Altnordischen, insbesondere auch in dem Lexicon Poeticum von F. Jónsson und in den Edda-Wörterbüchern findet.

Festzuhalten ist, dass in allen Fällen zur Zeit der ur- und altnordischen Sprachperiode der Begriff des Geheimnisvollen den geistig grundlegenden Kern aller Bedeutungsentfaltungen bildet. Gerade dieser Bedeutungskern fehlt aber, wie oben zu zeigen versucht wurde, dem finn. runo.

Auf altenglischer Seite kommt das Wort rün in den stark unter kirchlichem Einfluss stehenden Runeninschriften überhaupt noch nicht vor, und in den altenglischen Glossen treten nur Ableitungen wie gerýne 'Geheimnis', 'Sakrament', gerúna (= an. rúni) 'Berater' u.dgl. auf. Das Simplex rún begegnet erst in der geistlichen Dichtung, und zwar meist in der Bedeutung '(göttliches) Geheimnis'. Nur in der Dichtung "Daniel» ist rún auf die Flammenschrift in Belsazars Halle bezogen, so dass hier der Übergang von 'geheime Botschaft' zu 'geheime Schrift' zu beobachten ist. Eindeutig im Sinne von 'Runenschrift' findet sich rún zum ersten Male in der Merkdichtung des Exeterbuches: rúne writan, wobei rúne ein kollektiver Singular ist wie im Urnordischen. Zu der ganzen Frage vergleiche H.-G. Goetz, "Geschichte des Wortes rún (Rune) und seiner Ableitungen im Englischen" (Diss. Göttingen 1964).

Auf südgermanischem (deutschem) Boden bietet bereits die Fibel von Freilaubersheim (KJ 144) das Wort 'Rune' in der ersten Zeile der Inschrift: Bōso wraet rüna 'B. schrieb die Rune(n)', wobei der Akk. rüna formal Singular oder Plural sein kann, nach dem Muster des Gebrauchs von ags. rún (s.o.) wohl eher als kollektiver Singular zu betrachten ist.

In der mittelhochdeutschen Literatur bedeutet rûne vor allem 'Ge- 
heimnis', daneben 'geheime Beratung', 'Geflüster' u.ä. Bemerkenswert ist die Verbindung kein rede noch kein rune 'kein öffentliches und kein heimliches Gerede' in Konrad von Würzburgs Trojan. Krieg (vgl. Müller-Zarncke, Mhd. Wtb. II, 784 f.).

Der - soviel ich weiss - einzige Forscher, der eine Alternative zu der bisherigen Etymologie von runo wenigstens in Erwägung gezogen hat, ist $\mathrm{J}$. Weisweiler in einem kurzen Beitrag zu F. Altheims Buch »Literatur und Gesellschaft im ausgehenden Altertum" (1948), 289. Er verweist auf die Namen gewisser skaldischer Metren wie runhending, auch runhendingr háttr 'Reihenreim, reihenreimendes Metrum' zu runi m. oder runa f. 'Lauf, Bahn, Reihe' oder hrunhenda, Name eines bestimmten Preisliedes, hrynhendr háttr 'rauschend reimendes Metrum'. Trotzdem hält er selbst diese Herleitung für unsicher und die alte Anknüpfung des finnischen Wortes an germ. ${ }^{*} r u \bar{n} \bar{o}-$ für durchaus möglich.

Dabei war, wie mir scheint, Weisweiler bereits auf der richtigen Spur; nur die Anknüpfung an die Namen skaldischer Metren war allein schon vom historischen Standpunkt aus verfehlt. Zwar hatte er ein Grundwort an. runi oder runa bereits angesprochen, ohne aber diesen Faden weiter zu verfolgen.

Das Grundwort runa f. findet sich nun in der späturnordischen Inschrift (2. H. des 7. Jh.s) auf dem Blekinger Stein von Björketorp (KJ 97). Der die anschliessende Fluchformel einleitende Satz lautet, in Worte aufgelöst: haidR-rūnō ronu falahak haidera gina-rūnaR. 'Der Glanzrunen Reihe barg hier ich, Zauberrunen'. Hierin ist das Wort ronu der Akk. Sg. zu *rona 'Reihe', in diesem Fall also im Sinne von 'Aneinanderreihung von Worten in einer rhythmischen Folge'. Es ist ein Zufall, dass in dieser Inschrift das Wort für Reihe (urgerm. *runōn- bzw. *ronōn-1 ${ }^{1} \mathrm{zu}$ der in an. renna 'laufen' enthaltenen Wurzel $r e-n$-) unmittelbar neben dem etymologisch völlig verschiedenen Worte für 'Runen' steht.

1 Das Nebeneinander von klass.-urn. *ronō (an. *rona) und urn. *runō (neuisl. runa) 'Reihe' erklärt sich durch alten Systemausgleich des Wurzelvokals; vgl. etwa stufa : stofa 'Stube', uxi : oxi 'Ochse', altnorw. kuma : altisl. koma 'kommen', sunr : sonr 'Sohn', guð : goð 'Gott', gull : goll 'Gold'. 
Nun fehlt das entsprechende altnordische Wort *rona bzw. runa 'Reihe' in der altnordischen Literatur trotz ihres gewaltigen Umfangs vollständig. Dass es aber einstmals vorhanden war, wird durch neuisl. runa f. bezeugt. S. Blöndal gibt in seinem Wörterbuch dazu folgende Übersetzungen: 1. (röð) (uafbrudt) Rakke. - 2.a. (pula) Ramse. - b. (dala) uafbrudt Tale. - 3. (langur ás) lang Baklie (Nl.). - 4. (Breiðd.) = lön ['rechteckiger Heuhaufe']. - Árni Böðvarsson rerzeichnet in seiner 'Íslenzk Orðabók' (1963), 538 im wesentlichen die gleichen Bedeutungen von runa; nur fügt er noch die Bedeutung 'musikalische Suite' ${ }^{1}$ hinzu.

Man ersieht daraus, dass die Bedeutungsvarianten dieses Wortes urn. runō, neuisl. runa f. der Bedeutung von finn. runo nahezu gleichen. Das gilt insbesondere für die Verwendung dieses Wortes in der Inschrift von Björketorp und für die aus dem Grundbegriff 'Reihe' entwickelten Sonderbedeutungen von neuisl. runa 'ununterbrochene Reihe von Wörtern', 'pula', womit in der altisl. Dichtung eine Merkstrophe bezeichnet wird, die eine Reihe von Synonymen aufzählt. In allen Fällen handelt es sich - entsprechend dem finn. runo - um ein verhäItnismässig kurzes dichterisches Gebilde.

Formil kann man nun finn. runo entweder auf urgerm. ${ }^{*}$ runo $\bar{o}^{n}\left(-\bar{o}^{n}=\right.$ nasal. $\left.\bar{o}\right)$ oder auf urn. *runō zurückführen.

Zusammenfassend kann gesagt werden, dass bei der Annahme einer Entlehnung von finn. runo aus urgerm. ${ }^{*}$ runo $\bar{o}^{n}$ oder eher urn. *runō 'Reihe', 'Reihenfolge von Wörtern' sowohl formal wie bedeutungsmässig eine nahezu vollständige Übereinstimmung zwischen dem finnischen und dem germanischen Etymon besteht.

Wolfgang Krause

1 Auch das entsprechende Maskulinum runi findet sich als Simplex nur in den neuskandinavischen Sprachen, z.B. neuisl. runi 'Lauf', während altisl. -runi nur in komponierten Formen auftritt wie uppruni 'Wachstum, Ursprung', vegg-runi 'Dachtraufe'. 